\title{
Expression, production and release of the Eis protein by Mycobacterium tuberculosis during infection of macrophages and its effect on cytokine secretion
}

\author{
Linoj P. Samuel, ${ }^{1} \dagger$ Chang-Hwa Song, ${ }^{2}$ Jun Wei, ${ }^{1} \ddagger$ Esteban A. Roberts, ${ }^{1} \S$ \\ John L. Dahl, ${ }^{3}$ Clifton E. Barry, III, ${ }^{4}$ Eun-Kyeong Jo ${ }^{2}$ \\ and Richard L. Friedman ${ }^{1}$ \\ ${ }^{1}$ Department of Microbiology and Immunology, University of Arizona, Tucson, AZ 85724, USA \\ ${ }^{2}$ Department of Microbiology, College of Medicine, Chungnam National University, Daejeon \\ 301-747, South Korea \\ ${ }^{3}$ School of Molecular Bioscience, Washington State University, Pullman, WA 99164, USA \\ ${ }^{4}$ Tuberculosis Research Section, National Institute of Allergy and Infectious Disease, Rockville, \\ MD 20852, USA
}

Correspondence

Linoj P. Samuel

Linoj_Samuel@urmc.rochester.

edu

Received 18 September 2006

Revised 24 October 2006

Accepted 25 October 2006

\begin{abstract}
The eis gene of Mycobacterium tuberculosis has been shown to play a role in the survival of the avirulent Mycobacterium smegmatis within the macrophage. In vitro and in vivo analysis of $\Delta$ eis deletion mutants and complemented strains showed no effect on survival of $M$. tuberculosis in U-937 macrophages or in a mouse aerosol infection model, respectively. Further studies were done in an attempt to determine the role of eis in M. tuberculosis intracellular survival and to define a phenotypic difference between wild-type and the $\Delta$ eis deletion mutant. Bioinformatic analysis indicated that Eis is an acetyltransferase of the GCN5-related family of $N$-acetyltransferases. Immunofluorescence microscopy and Western blot analysis studies demonstrated that Eis is released into the cytoplasm of $M$. tuberculosis-infected U-937 macrophages. Eis was also found in the extravesicular fraction and culture supernatant of $M$. tuberculosis-infected macrophages. The effect of Eis on human macrophage cytokine secretion was also examined. Eis modulated the secretion of IL-10 and TNF- $\alpha$ by primary human monocytes in response both to infection with $M$. tuberculosis and to stimulation with recombinant Eis protein. These results suggest that Eis is a mycobacterial effector that is released into the host cell to modulate inflammatory responses, possibly via transcriptional or post-translational means.
\end{abstract}

\section{INTRODUCTION}

Mycobacterium tuberculosis, the causative agent of tuberculosis (TB), is one of the oldest pathogens known to man and is responsible for the deaths of over 1.8 million people every year (Corbett et al., 2003). M. tuberculosis survives within the host macrophage by arresting the normal development of the phagosome (Chan et al., 1991; Fratti et al., 2003).

†Present address: Department of Clinical Microbiology, The University of Rochester, 601 Elmwood Ave, Box 710, Rochester, NY 14642, USA.

łPresent address: Amgen Inc., Thousand Oaks, CA 91320, USA.

§resent address: Department of Molecular Genetics and Microbiology, University of New Mexico, Albuquerque, NM 87131, USA.

Abbreviations: 3D-PSSM, three-dimensional position-specific scoring matrix; FCS, fetal calf serum; GNAT, GCN5-related N-acetyltransferase; $I F$, immunofluorescence microscopy; IL, interleukin; PPD, purified protein derivative; TB, tuberculosis; TNF- $\alpha$, tumour necrosis factor alpha.
Phagosome-lysosome fusion is inhibited in part by effectors that are secreted by M. tuberculosis, such as mannose-capped lipoarabinomannan (ManLAM) (Chan et al., 1991; Fratti et al., 2003). In this manner, the bacterium is able to avoid the toxic effects of lysosomal enzymes and the normal acidified environment of a mature phagosome.

However, mycobacterial survival also hinges on its ability to modulate host cytokine secretion. M. tuberculosis infection or mycobacterial proteins alone induce the secretion of a large number of cytokines including interleukin (IL)-1, IL-2, IL-10, IL-12 and tumour necrosis factor alpha (TNF- $\alpha$ ) by monocytes/macrophages (Barnes et al., 1992; Fulton et al., 1996; Lee et al., 2003b; Wallis et al., 1990). The important proinflammatory cytokine TNF- $\alpha$ plays a key role in the host immune defence against TB (Flynn et al., 1995; Keane et al., 2001). In contrast, IL-10 downregulates cytokine production and accessory cell function of mononuclear phagocytes 
(D'Andrea et al., 1993; de Waal Malefyt et al., 1991) and its expression is also induced by various mycobacterial products (Barnes et al., 1992).

A wide variety of mycobacterial proteins and lipids are secreted into the cytoplasm of $M$. tuberculosis-infected macrophages (Beatty \& Russell, 2000; Beatty et al., 2001, 2000; Xu et al., 1994). The trafficking of these mycobacterial components within the endocytic network of the host cell may play a role in modulating macrophage function to the advantage of the bacterium. These mycobacterial components are also released from the macrophage into the extracellular milieu in vesicular compartments (Beatty et al., 2001; Rhoades et al., 2003). Furthermore, these vesicles are taken up by uninfected bystander cells (Beatty et al., 2001, 2000). The propensity of M. tuberculosis to extend its influence beyond the constraints of the infected host cell is in keeping with its ability to subvert the immune system of the host to enhance its survival.

The eis (enhanced intracellular survival) gene of $M$. tuberculosis is only found in members of the M. tuberculosis complex and has been shown to confer enhanced survival on Mycobacterium smegmatis in U-937 macrophages and human macrophages (Wei et al., 2000). The Eis protein is a $42 \mathrm{kDa}$ protein that is secreted when M. tuberculosis is grown in liquid culture (Dahl et al., 2001). In the present study, we constructed and utilized an eis mutant $(\Delta e i s)$ and complemented strains of $M$. tuberculosis $\mathrm{H} 37 \mathrm{Ra}$ and $\mathrm{H} 37 \mathrm{Rv}$ to study the role the gene plays in the microbe's interactions with macrophages. We discovered that the Eis protein is produced and released by $M$. tuberculosis into the cytoplasm of the phagocyte during intracellular infection. We also found that Eis is able to modulate the secretion by human monocytes of the cytokines TNF- $\alpha$ and IL-10, which play a major role in the regulation of the inflammatory response to infection. Bioinformatic studies show that Eis is a putative acetyltransferase of the GCN5-related family of $\mathrm{N}$-acetyltransferases (GNAT) that may play a role in deregulating macrophage cytokine production.

\section{METHODS}

Strains and growth media. M. tuberculosis $\mathrm{H} 37 \mathrm{Rv}$ and $\mathrm{H} 37 \mathrm{Ra}$ were grown in Middlebrook 7H9 (Difco) broth. For growth on solid culture media, Middlebrook 7H10 (Difco) with 10\% OADC was used. Mycobacterial inocula for infection of U-937 cells with $\mathrm{MtbRa} / g f p$ and $\mathrm{MtbRa} \Delta e i s / g f p$ were prepared as previously described (Wei et al., 2000). For growing Escherichia coli transformants, LuriaBertani (LB) broth or agar with $200 \mu \mathrm{g}$ hygromycin $\mathrm{B} \mathrm{ml} \mathrm{ml}^{-1}$ or $100 \mu \mathrm{g}$ ampicillin $\mathrm{ml}^{-1}$ was used.

M. tuberculosis $\mathrm{H} 37 \mathrm{Ra}$ wild-type and $\Delta$ eis were transformed with the vector $\mathrm{pBEN}$, which contains $g f p$ driven by $\mathrm{p} h \mathrm{sp} 60$, a strong heat-shock promoter from Mycobacterium bovis BCG (Table 1) (Saviola et al., $2003)$ to generate the $g f p$-expressing strains MtbRa/ $g f p$ and MtbRa $\Delta$ eis/ $g f p$, respectively. The promoterless $g f p$ vector $p F P V 27$ was used as the negative control for these studies. Both vectors were generously supplied by Stanley Falkow, Stanford University School of Medicine. Bacteria were grown on Middlebrook 7H10 medium containing $25 \mu \mathrm{g}$ kanamycin $\mathrm{ml}^{-1}$. Fluorescence of transformants was confirmed by fluorescence microscopy, as described below.

Human macrophage-like U-937 cells (ATCC; 1593.2 CRL) were grown in RPMI 1640 medium (Sigma) containing $10 \%$ fetal calf serum (FCS) (Atlanta Biologicals) under $5 \% \mathrm{CO}_{2}$ at $37^{\circ} \mathrm{C}$ (Nilsson \& Sundstrom, 1974). U-937 macrophage monolayers were prepared by transformation of cells in T75 flasks (Corning) using phorbol myristic acetate (PMA), as previously described (Wei et al., 2000).

General DNA methods. For DNA manipulations, standard protocols were followed (Sambrook et al., 1989). Agarose was purchased from Promega. Restriction enzymes, Klenow fragment of T4 DNA polymerase, T4 DNA ligase and calf intestinal alkaline phosphatase (CIAP) were from New England BioLabs or Gibco-BRL Life Technologies. All reagents were used according to the manufacturer's instructions. Plasmids were constructed in E. coli HB101 or DH5a cells and prepared using a Qiaprep spin miniprep kit (Qiagen).

Table 1. Plasmids used in this study

\begin{tabular}{|c|c|c|}
\hline Plasmid & Relevant characteristics & Reference or source \\
\hline pMV306 & Mycobacterial integrating vector, integrates into the att $\mathrm{B}$ site, $\mathrm{Kan}^{\mathrm{r}}$ & Stover et al. (1991) \\
\hline pSP72 & E. coli cloning vector, $\mathrm{Amp}^{\mathrm{r}}$ & Promega \\
\hline pRL498 & pUC-based vector, containing $1.3 \mathrm{~kb}$ hygromycin resistance cassette, $\mathrm{Hyg}^{\mathrm{r}} \mathrm{Kan}^{\mathrm{r}}$ & C. E. Barry, III* \\
\hline pMJ10 & ts oriM; sacB conterselection, $\operatorname{Kan}^{\mathrm{r}} \mathrm{Gent}^{\mathrm{r}}$ & Pelicic et al. (1997) \\
\hline p69 & pOLYG with $2.99 \mathrm{~kb} M$. tuberculosis genomic DNA fragment containing the eis gene & Wei et al. (2000) \\
\hline p6237 & $\begin{array}{l}1.3 \text { kb hygromycin gene released by XbaI from pRL498 replacing a } 367 \text { bp internal NheI } \\
\text { fragment from p } 6297\end{array}$ & This study \\
\hline p6297 & pSP72 with $1.6 \mathrm{~kb}$ ApoI-PvuI eis-containing fragment from pOLYG & This study \\
\hline p6301 & pMV306 with $1.6 \mathrm{~kb}$ ClaI-HindIII eis-containing fragment from p6297 & This study \\
\hline p6308 & pMJ10 with $2.5 \mathrm{~kb} \mathrm{XbaI-BamHI}$ fragment from p6237 containing eis disrupted by the $h y g$ gene & This study \\
\hline pBEN & $g f p$ driven by $\mathrm{p} h s p 60$ from $M$. bovis BCG & Saviola et al. (2003) \\
\hline pFPV27 & Promoterless $g f p$ reporter vector & Barker et al. (1998) \\
\hline pET-15b & Carries N-terminal His tag & Novagen \\
\hline pET-15b-eis & pET vector with eis fragment & This study \\
\hline
\end{tabular}

${ }^{\star}$ National Institutes of Health. 
Recombinant Eis. In order to obtain purified Eis protein in sufficient quantities for use in our studies, we constructed a recombinant His-tagged Eis protein. The $1.3 \mathrm{~kb}$ eis gene was PCR amplified and cloned into the pET-15b vector (Novagen) so as to obtain a recombinant protein with an N-terminal His-tag. The plasmid bearing the insert (pET15b-eis) was transformed into the E. coli expression strain BL-21 DE-3 pLysS (Novagen) (Table 2). Recombinant protein was then induced, harvested and purified as per the manufacturer's protocol (Novagen). The one step Ni-NTA purification process resulted in a single protein band, of molecular mass similar to that predicted for Eis (42 kDa) (Wei et al., 2000). Purity of the recombinant Eis was greater than $99 \%$, as determined by scanning densitometry analysis of SDS-PAGE gels, and the His-tagged Eis protein also reacted with the anti-Eis antibody (data not shown). Limulus lysate amoebocyte assay was utilized to test for endotoxin contamination.

Infection of mice. Five- to eight-week-old female C57/BL6 mice were purchased from Taconic and aerosol infected as described previously (Boshoff et al., 2003). Each mouse was infected with approximately 50 c.f.u. $M$. tuberculosis and bacterial burdens were monitored by plating of lung and spleen homogenates from four or five mice at different times during the infection. In addition, mice lungs and spleens were fixed in $10 \%$ neutral buffered formalin, embedded in paraffin, sectioned, and stained for histology with either haematoxylin/eosin or Ziehl-Neelsen stain.

Immunofluorescence microscopy of infected U-937 macrophages. One-millilitre aliquots of RPMI containing $2 \times 10^{5} \mathrm{U}-937$ cells were plated into each well of a 24 -well tissue culture plate (Falcon) containing sterile glass coverslips. Cells were infected the next day as described previously (Wei et al., 2000) with MtbRa/gfp and $\mathrm{MtbRa} \Delta e i s / g f p$ at an m.o.i. of 20. At appropriate time points, coverslips were washed three times with PBS and cells fixed by addition of $1 \mathrm{ml} 3 \%$ formaldehyde in PBS to each well. After $15 \mathrm{~min}$, coverslips were washed three times with PBS and cells were permeabilized by addition of $0.1 \%$ Triton X-100 for $5 \mathrm{~min}$. Coverslips were again washed, and incubated with a 1:500 dilution of anti-Eis antibody for $1 \mathrm{~h}$ at room temperature on an orbital shaker. Anti-Eis antibody is an affinity-purified antibody to Eis generated in rabbits as described previously (Dahl et al., 2001). Alexafluor 594 goat antirabbit IgG linked to a fluorescent red dye (Molecular Probes;
1:10000) in PBS containing 10\% FCS was then added and incubated with the coverslips. Wells were washed and coverslips were removed to a paper towel to air dry in the dark. Two microlitres of $p$-phenylenediamine mounting medium was added to each coverslip, which was then inverted onto a glass slide, sealed with clear nail polish and viewed using a Nikon Eclipse 2000-S fluorescent/phasecontrast microscope. Images were obtained as TIFF files using Metacam software and adjusted for consistent contrast using Photoshop 7.0 (Adobe).

\begin{abstract}
Infection, harvesting and fractionation of infected U-937 macrophage monolayers for Western blot analysis of macrophage cytoplasm. Infection of U-937 monolayers was carried out as described above (Wei et al., 2000). An m.o.i. of 15 was used and the infection was allowed to proceed for $4 \mathrm{~h}$. Monolayers were then washed and $15 \mathrm{ml} \mathrm{RPMI}+5 \%$ human $\mathrm{AB}$ sera was added to each flask and incubated for $48 \mathrm{~h}$. Monolayers were then washed with Hanks' balanced salts solution (HBSS) and harvested into $15 \mathrm{ml}$ HBSS by agitation with $3 \mathrm{~mm}$ glass beads. Cells were washed and resuspended in cold homogenization buffer $(250 \mathrm{mM}$ sucrose, $0.5 \mathrm{mM}$ EDTA, $0.5 \mathrm{mM}$ EGTA, $20 \mathrm{mM}$ HEPES) and then lysed by repeated passage through a 25 gauge needle. Lysate was centrifuged at $300 \mathrm{~g}$ for $10 \mathrm{~min}$ followed by two subsequent spins at $100 \mathrm{~g}$ for 5 min each. The supernatant was carefully layered over a $12 \%$ sucrose cushion and centrifuged at $800 \mathrm{~g}$ for $45 \mathrm{~min}$ at $4{ }^{\circ} \mathrm{C}$. The cytoplasmic fraction remained in a distinct upper layer, separate from mycobacteria and phagosomes containing mycobacteria in the lower layer and pellet, respectively.
\end{abstract}

Isolation of exocytic vesicles from culture supernatant of infected U-937 macrophages. Exocytic vesicles were isolated from tissue culture supernatants as described previously (Beatty et al., 2001). Briefly, tissue culture supernatants from U-937 macrophages infected with $M$. tuberculosis H37Ra were harvested and subjected to a series of centrifugation steps to remove whole cells and bacteria. The supernatant was then centrifuged for $60 \mathrm{~min}$ at $100000 \mathrm{~g}$. The resulting pellet contained the vesicles released from the macrophages which were analysed by Western blotting for the presence of the Eis protein. Culture supernatant obtained after

Table 2. Bacterial strains used in this study

\begin{tabular}{|c|c|c|}
\hline Strain & Description & Reference or source \\
\hline \multicolumn{3}{|l|}{ E. coli } \\
\hline DH5 $\alpha$ & supE44 $\Delta$ lacU169 ( $\phi 80$ lacZ $\Delta \mathrm{M} 15)$ hsdR17 recA1 endA1 gyrA96 thi-1 relA1 & Gibco-BRL \\
\hline \multicolumn{3}{|l|}{ M. tuberculosis } \\
\hline $\mathrm{H} 37 \mathrm{Ra}$ & Attenuated $\mathrm{H} 37 \mathrm{Rv}$ & D. Young* \\
\hline $\mathrm{H} 37 \mathrm{Rv}$ & Virulent laboratory strain (ATCC 27294) & $\mathrm{CSU} \dagger$ \\
\hline $\mathrm{H} 37 \mathrm{Rv} \Delta$ eis & Double-crossover recombinant of $\mathrm{H} 37 \mathrm{Rv}$ with $\mathrm{p} 6308, \Delta e i s:: h y g, \mathrm{Hgr}^{\mathrm{r}}$ & This study \\
\hline $\mathrm{H} 37 \mathrm{Rv}$ eis complemented & $\begin{array}{l}\text { H37Rv containing integrated copy of p6308 and p6301 } \Delta e i s:: h y g, \text { eis::attB, } \\
\mathrm{Hgr}^{\mathrm{r}}, \mathrm{Kan}^{\mathrm{r}}\end{array}$ & This study \\
\hline
\end{tabular}

${ }^{\star}$ Imperial College School of Medicine, London, England.

$\dagger$ Colorado State University. 
processing as described above was concentrated threefold using YM10 Centriprep filters (Millipore) and analysed by Western blot for the presence of Eis protein.

Western blot analysis. Sample protein concentrations were determined by BCA protein assay (Pierce). Samples were separated on $10 \%$ SDS-PAGE gels along with prestained molecular mass standards (Gibco-BRL). Electrophoresis was performed at $200 \mathrm{~V}$ and $80 \mathrm{~mA}$ for $3-4 \mathrm{~h}$. Proteins were transblotted for $1 \mathrm{~h}$ using MiniProtean 3 cells (Bio-Rad) onto PVDF membranes (Millipore) which were then blocked with $5 \%$ skim milk in PBS containing $0.02 \%$ sodium azide. Membranes were incubated with primary antibody in PBS containing $0.25 \%$ powdered skim milk for $1 \mathrm{~h}$, washed three times with PBS and then probed with secondary antibody for $1 \mathrm{~h}$. Secondary antibody was goat anti-rabbit IgG conjugated to horseradish peroxidase (HRP) diluted 1:20000 and used in blots. Membranes were then washed three times with PBS and incubated with chemiluminescent substrate for $5 \mathrm{~min}$ followed by exposure to $\mathrm{X}$-ray film for appropriate periods of time. All reagents for development of the chemiluminescent Western blots were obtained from Pierce.

Isolation and cultivation of human monocytes for analysis of cytokine secretion in response to antigenic stimulation. Venous blood was drawn from tuberculin-positive healthy subjects into sterile blood collection tubes, and peripheral blood mononuclear cells (PBMCs) were isolated by density sedimentation over Histopaque-1077 (Sigma). PBMCs were suspended at a density of $2 \times 10^{6}$ cells $\mathrm{ml}^{-1}$ in complete RPMI medium (Gibco-BRL) with $10 \%$ fetal bovine serum (Gibco-BRL), sodium pyruvate, nonessential amino acids, penicillin $\mathrm{G}\left(100 \mathrm{IU} \mathrm{ml}^{-1}\right)$ and streptomycin $\left(100 \mu \mathrm{g} \mathrm{ml}^{-1}\right)$. Cells were incubated for $1 \mathrm{~h}$ at $37^{\circ} \mathrm{C}$ and non-adherent cells were removed by pipetting off the supernatant. Adherent monocytes were collected as previously described (Song et al., 2003). The recovered cells were $>95 \% \mathrm{CD}^{+} 4^{+}$cells, as determined by flow cytometry using an anti-CD14 antibody. The cells were then stimulated with either purified protein derivative (PPD) (Statens Serum Institut), Eis, or lipopolysaccharide (LPS, $10 \mathrm{ng} \mathrm{ml}^{-1}$; Sigma) and incubated at $37^{\circ} \mathrm{C}$ in a $5 \% \mathrm{CO}_{2}$ humidified air atmosphere. The supernatants from $18 \mathrm{~h}$ (for TNF- $\alpha$ ) and $48 \mathrm{~h}$ (for IL-10) cultures were frozen at $-80^{\circ} \mathrm{C}$ until used in ELISA.

Preparation of inocula and infection of human monocytes with $M$. tuberculosis for analysis of cytokine secretion. $M$. tuberculosis $\mathrm{H} 37 \mathrm{Rv}$ was grown to late exponential phase in Middlebrook $7 \mathrm{H} 9$ as described previously, aliquoted and stored at $-70{ }^{\circ} \mathrm{C}$. Representative vials were thawed and c.f.u. enumerated on Middlebrook 7H10 agar. Single-cell suspensions of mycobacteria were obtained by a modification of standard methods. Briefly, aliquots of frozen $M$. tuberculosis were cultured in $7 \mathrm{H} 9$ broth with $0.5 \%(\mathrm{v} / \mathrm{v})$ glycerol at $37^{\circ} \mathrm{C}$ in $5 \% \mathrm{CO}_{2}$ for $7-10$ days so that cultures reached mid-exponential growth phase. Bacterial cultures were pelleted at $3000 \mathrm{~g}$ for $10 \mathrm{~min}$ and resuspended in $7 \mathrm{H} 9$ broth. Clumped mycobacteria were dispersed with an ultrasonic cell disrupter (3-5 min, $35 \mathrm{kHz}$; Bandelin). Bacteria were then resuspended in $1 \mathrm{ml}$ RPMI 1640 medium and clumps were disrupted by multiple passages through a 25 gauge needle. Mycobacterial viability, as assessed by the number of c.f.u., was $60-70 \%$.

Adherent human monocytes were washed three times with $\mathrm{Ca}^{2+}$ - and $\mathrm{Mg}^{2+}$-free PBS, and adherent monolayers were replenished with complete medium without antibiotics. The cells were incubated overnight without stimulation at $37^{\circ} \mathrm{C}$ in $5 \% \mathrm{CO}_{2}$. After overnight incubation, monocytes $\left(2 \times 10^{5} \mathrm{ml}^{-1}\right)$ were infected with mycobacteria using an m.o.i. of 1 for either $18 \mathrm{~h}$ or $48 \mathrm{~h}$, and the supernatants were then recovered for ELISA determination of cytokine levels of TNF- $\alpha$ and IL-10, respectively.
ELISA. ELISA was used to detect TNF- $\alpha$ and IL-10 levels (BD Biosciences) in monocyte culture supernatants, as previously described (Lee et al., 2003a). Assays were performed as recommended by the manufacturers. Cytokine concentrations in the samples were calculated using standard curves generated from recombinant cytokines, and the results were expressed in picograms per millilitre. The difference between triplicate wells was consistently less than $10 \%$ of the mean.

Bioinformatic analysis. Protein sequences for analysis were obtained in FASTA format and input into respective programs at the interface locations described below. Reverse position-specific (RPS)BLAST is a variation of PSI-BLAST, which utilizes the Conserved Domain Database (CDD) to compare a single sequence against a database of PSSM (Position-Specific Score Matrix) models (Marchler-Bauer \& Bryant, 2004). This is available for use at http:// www.ncbi.nlm.nih.gov/Structure/cdd/wrpsb.cgi. Pfam is a comprehensive database of protein domain families based on seed alignments using hidden Markov model profiles. The 3D-PSSM (threedimensional position-specific scoring matrix) server is designed to take a protein sequence of interest and attempt to predict its $3 \mathrm{D}$ structure and its probable function. This program is able to confidently predict structural and functional relationships better than standard local alignment search tools such as PSI-BLAST (Kelley et al., 2000). 3D-PSSM is administered by Lawrence Kelley and is available for use at http://www.sbg.bio.ic.ac.uk/servers/3dpssm/.

Statistical analysis. For statistical analysis, data obtained from independent experiments were presented as the mean \pm SD and were compared by Student's $t$-test or for multiple comparisons by ANOVA. Differences were considered significant for $P<0.05$.

\section{RESULTS}

\section{Survival of M. tuberculosis wild-type and eis mutant in vitro and in vivo}

Having demonstrated previously that the eis gene enhanced intracellular survival of M. smegmatis (Wei et al., 2000), it was only natural to look for a similar phenotype in $M$. tuberculosis. The first step towards answering this question was to develop a $\Delta e i s$ and complemented strain in both $M$. tuberculosis H37Ra and H37Rv. Deletion and complementation of the eis gene was confirmed by both Southern and Western blot analysis (data not shown). Survival of the $\mathrm{H} 37 \mathrm{Rv}$ wild-type, $\Delta$ eis mutant and complemented strains in U-937 macrophage monolayers was then compared. There was no significant difference between the three strains in terms of intracellular survival in U-937 macrophages for the duration of the 7 day experiment (data not shown).

Survival of these three strains was then compared in an in vivo mouse aerosol model (Dahl et al., 2003). Six- to eightweek-old female C57BL/6 mice were infected via the aerosol route with $M$. tuberculosis $\mathrm{H} 37 \mathrm{Rv}$ wild-type, $\Delta$ eis mutant and complemented strains. At various time points over a 17 week period, the mice were sacrificed and lung and spleen homogenates were plated for c.f.u. determinations and tissue samples were also taken for histological evaluation. There appeared to be no significant difference between survival of the wild-type, $\Delta$ eis mutant and complemented strains in the in vivo model as determined by viable plate counts (Fig. 1). Also, no histological 


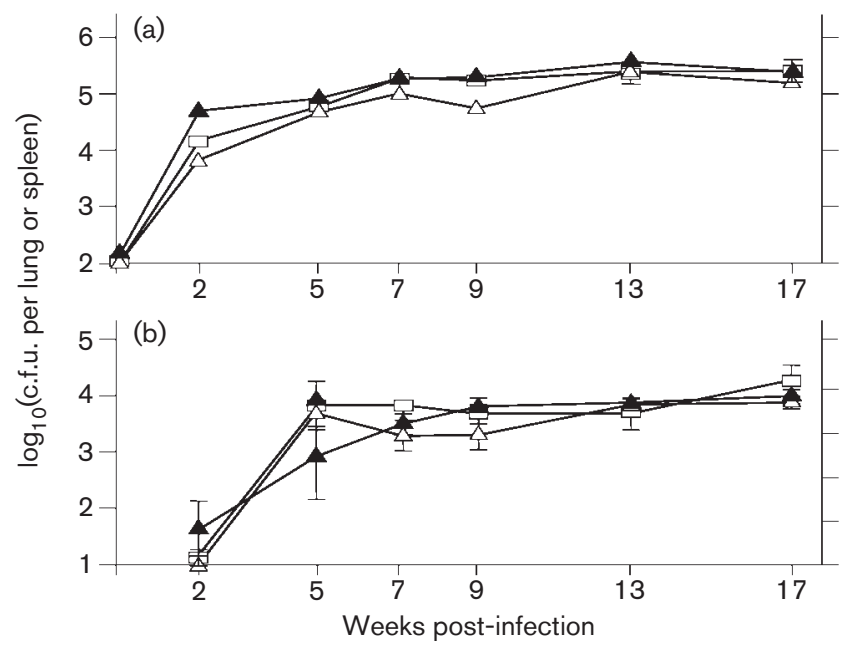

Fig. 1. Growth of M. tuberculosis H37Rv, $\Delta$ eis and complemented strains in the lungs (a) and spleens (b) of aerosolinfected mice. Female C57/BL6 mice were infected via the aerosol route and monitored by c.f.u. determinations of lung and spleen homogenates via viable plate counts. These data are representative of three independent experiments using four or five mice per time point after initial infection. $\square$, Wild-type; $\triangle, \Delta$ eis; $\boldsymbol{\Lambda}$, complemented $\Delta$ eis.

differences were observed between infected lung or spleens from wild-type or $\Delta$ eis mutants. This included severity of granuloma formation and inflammation, granuloma numbers and numbers of acid-fast bacteria per tissue section (data not shown).

\section{Bioinformatic analysis of Eis}

Bioinformatic analysis of the Eis protein sequence using the protein-protein BLAST (BLASTP) program (Altschul et al., 1990) yielded a large number of homologous proteins, many of which were members of the GNAT superfamily of proteins. RPS-BLAST (Marchler-Bauer \& Bryant, 2004) analysis of Eis revealed the presence of an acetyltransferase domain that is characteristic of the pfam00583 family of
GNAT acetyltransferases (Neuwald \& Landsman, 1997). The acetyltransferase domain extends from residues 61 to 137 of the 402 aa Eis protein sequence and contains the conserved residues (V/I-X-X-X-X-Q/R-X-X-G-X-G/A) that are characteristic of this domain (Neuwald \& Landsman, 1997). A substitution of glycine or alanine with lysine at the last conserved residue, as seen with Eis, is found in other members of this family (Neuwald \& Landsman, 1997). Since members of the GNAT superfamily have poor sequence homology but share common 3D structural features (Dyda et al., 2000), the 3D-PSSM program was used to search for proteins showing significant fold compatibility with Eis. Fold analysis of a 154 aa section of the Eis sequence, which included the acetyltransferase domain using 3D-PSSM, indicates that Eis shares strong similarities with several proteins belonging to the GNAT family of acetyltransferases including the conserved residues (Table 3). These bioinformatics analyses suggest that Eis may function as an acetyltransferase.

\section{Presence of Eis in the cytoplasm of macrophages infected with $M$. tuberculosis}

Because the Eis protein was shown to be secreted into the bacterial culture medium by $M$. tuberculosis in vitro (Dahl et al., 2001), studies were done to examine infected macrophages for the presence of the Eis protein in the host cell cytoplasm. M. tuberculosis H37Ra and $\Delta$ eis strains were transformed with the pBEN plasmid, which contains the green fluorescent protein $(g f p)$ gene driven by $\mathrm{p} h \mathrm{sp} 60$ from Mycobacterium bovis BCG (Saviola et al., 2003). M. tuberculosis $\mathrm{H} 37 \mathrm{Ra}$ wild-type and $\Delta$ eis strains expressing $g f p$ were named $\mathrm{MtbRa} / g f p$ and $\mathrm{MtbRa} \Delta$ eis/gfp, respectively. The MtbRa/gfp- and MtbRa $\Delta e i s / g f p$-infected cells were washed, permeabilized, and probed with rabbit anti-Eis antibody (Dahl et al., 2001) followed by goat anti-rabbit antibody labelled with a fluorescent red dye.

Macrophages infected with MtbRa $\Delta e i s / g f p$, probed with both primary and secondary antibodies and visualized using immunofluorescence microscopy (IF) showed no red staining for the Eis protein at any time point due to the absence of Eis production in the MtbRa $\Delta$ eis/gfp mutant

Table 3. 3D-PSSM search for structural homologues to the Eis protein

The predicted 3D structure of Eis shows significant homology to the known 3D structures of several members of the pfam00583 family of GNAT acetyltransferases as demonstrated by their E-values. The 3D structures of the proteins with the lowest E-values demonstrate the highest levels of similarity to the predicted $3 \mathrm{D}$ structure of Eis.

\begin{tabular}{|llll|}
\hline Protein & \multicolumn{1}{c|}{ Organism } & \multicolumn{1}{c|}{ Family } \\
\hline Aminoglycoside $2^{\prime}$-N-acetyltransferase & M. tuberculosis & Predicted GNAT & Galue \\
Aminoglycoside 3 - $N$-acetyltransferase & Serratia marcescens & GNAT & $3.61 \times 10^{-3}$ \\
Histone acetyltransferase & Saccharomyces cerevisiae & GNAT & $9.56 \times 10^{-3}$ \\
Aminoglycoside $6^{\prime}-N$-acetyltransferase & Enterococcus faecium & GNAT & $1.09 \times 10^{-2}$ \\
Phosphinothricin $N$-acetyltransferase & Bacillus subtilis & Predicted GNAT & $2.12 \times 10^{-2}$ \\
Protein Yjcf & B. subtilis & GNAT & $3.29 \times 10^{-2}$ \\
& & & $1.71 \times 10^{-1}$ \\
\hline
\end{tabular}


(Fig. 2a, b). Starting at $4 \mathrm{~h}$ post-infection up to $96 \mathrm{~h}$ postinfection, U-937 cells infected with MtbRa/gfp demonstrated the presence of Eis protein in the cytoplasm as evidenced by red fluorescence when viewed by IF (Fig. 2ch). No Eis protein was visible when the cells were examined $30 \mathrm{~min}$ post-infection (data not shown). In many instances, macrophages appeared to contain Eis protein in the absence of visible infecting bacteria (Fig. 2f, g; arrowheads denote uninfected macrophages). In control experiments, no IF was observed in uninfected U-937 cells that were probed in a similar manner. This was also true for cells that were not permeabilized before the addition of the primary and secondary antibodies (data not shown), indicating that the Eis protein being visualized was not tethered to the surface of the macrophage. This experiment was repeated three times and similar results were obtained each time. Similar results were also observed using both J774 murine macrophages and primary human monocytes (data not shown).

\section{Western blot analysis of the cytoplasm of infected macrophages for the presence of Eis}

Western blot analysis using the anti-Eis antibody of lysates prepared from infected macrophages as previously described (Chakraborty et al., 1994) was used to confirm the IF observation of the presence of Eis in the cytoplasm of these macrophages. The Eis protein was not detected in the cytoplasmic fraction of macrophages infected with $M$. tuberculosis $\mathrm{H} 37 \mathrm{Ra} \Delta$ eis (Fig. 3, lane 1) but was found in the cytoplasmic fraction of U-937 cells infected with $M$. tuberculosis $\mathrm{H} 37 \mathrm{Ra}$ wild-type at $48 \mathrm{~h}$ after infection (Fig. 3, lane 2). The infecting bacteria had been removed from these fractions by centrifugation of the lysate over a $12 \%$ sucrose solution. To discount the possibility that the presence of Eis in the macrophage cytoplasm may be due to intracellular lysis of phagocytosed bacteria, the cytoplasmic fraction was probed by Western blot analysis for the presence of the $16 \mathrm{kDa}$ alpha crystallin protein (Acr) at $48 \mathrm{~h}$ after infection. Acr is a mycobacterial protein encoded by $h s p X$ that is upregulated in $M$. tuberculosis during infection of macrophages and is known not to be secreted from the bacteria (Dubnau et al., 2002). Acr was not detected in the cytoplasmic fraction of macrophages infected with $M$. tuberculosis by Western blot analysis.

We then attempted to explain the presence of Eis protein in macrophages that appeared to be visibly uninfected (Fig. $2 \mathrm{f}$ and $g$, arrowheads) by examining the vesicular fraction of the culture supernatant as described previously by Beatty


Fig. 2. Immunofluorescence microscopy demonstrating the presence of Eis in the cytoplasm of U-937 macrophages infected with MtbRa/gfp. U-937 macrophages were infected at an m.o.i. of 20 and at various time points cells were fixed, permeabilized, probed and viewed by IF. Mycobacteria appear as fluorescent green clumps while the Eis protein appears as fluorescent red dispersed throughout the cytoplasm of MtbRa/gfp-infected macrophages. (a) IF showing fluorescent green MtbRa_eis/gfp (white arrow) in U-937 macrophages. No Eis was detected in the cytoplasm of these macrophages as observed by the absence of red fluorescence. (b) Phase-contrast and fluorescent microscopy images of the field in (a) were merged, visualizing the macrophage and the fluorescent green bacteria (white arrow). (c) U-937 cells infected with MtbRa/gfp demonstrating red fluorescence throughout the cytoplasm due to the release of Eis protein from mycobacterial phagosomes at $4 \mathrm{~h},(\mathrm{~d}) 12 \mathrm{~h},(\mathrm{e}) 24 \mathrm{~h},(\mathrm{f}) 48 \mathrm{~h},(\mathrm{~g}) 72 \mathrm{~h}$ and (h) at $96 \mathrm{~h}$ after infection. In both (f) and (g) uninfected macrophages (indicated by white arrowheads) demonstrate the presence of the Eis protein in the cytoplasm in the absence of infecting bacteria. Bar, $20 \mu \mathrm{m}$. 


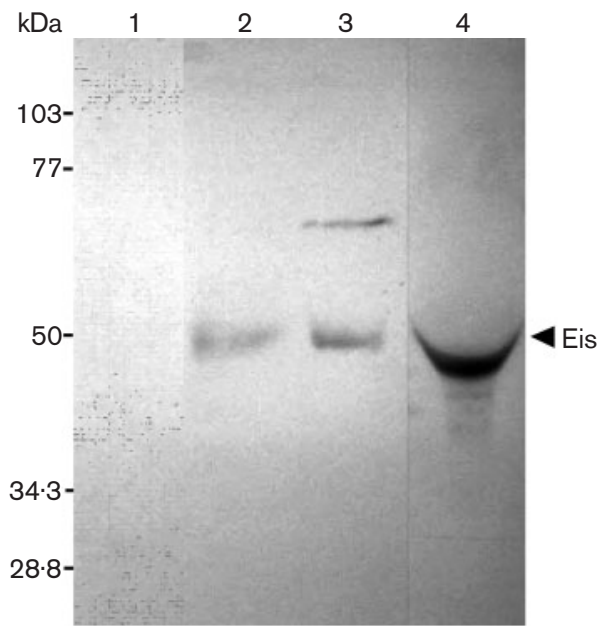

Fig. 3. Western blot detection of Eis in the cytoplasm and tissue culture supernatant of U-937 macrophages infected with M. tuberculosis H37Ra wild-type and $\Delta$ eis mutant. Molecular mass markers and their sizes are represented at the left side of the blot. All lanes were probed with anti-Eis antibody. Lane 1, cytoplasmic fraction of U-937 macrophages infected with $M$. tuberculosis H37RaAeis. No Eis protein is detected. Lane 2, presence of Eis in the cytoplasmic fraction of U-937 macrophages infected with M. tuberculosis H37Ra wild-type. Lane 3, extravesicular fraction from the tissue culture supernatant of $M$. tuberculosis H37Ra wild-type-infected U-937 macrophages. Eis was found to be associated with vesicles released from infected U-937 macrophages. Lane 4, presence of native Eis protein in the tissue culture supernatant of U-937 macrophages infected with $M$. tuberculosis H37Ra after removal of all vesicular material followed by concentration. In all cases, samples were loaded onto $10 \%$ SDS-PAGE gels, separated by electrophoresis, and transblotted onto PVDF membranes for Western blot analysis. Two hundred micrograms of total protein was loaded per lane. The black arrowhead to the right of the blot denotes the position of the Eis protein.

et al. (2000). Tissue culture supernatant from U-937 cells infected with M. tuberculosis H37Ra was harvested at $48 \mathrm{~h}$ after infection and then subjected to a series of centrifugation steps to remove any contaminating bacteria and macrophage organelles and to separate out the microvesicular fraction. Analysis of this fraction containing the vesicles released by macrophages into the culture supernatant by Western blot detected the presence of the Eis protein (Fig. 3, lane 3). The $75 \mathrm{kDa}$ band that is seen in this lane was not detected in either the cytoplasmic fraction (Fig. 3, lane 1) or the extravesicular fraction of U-937 cells infected with $M$. tuberculosis $\mathrm{H} 37 \mathrm{Ra} \Delta$ eis (data not shown). As the anti-Eis antibody used in these studies is affinity purified (Dahl et al., 2001), it is unlikely to cross-react with host cell proteins. Eis protein has a tendency to aggregate in solution and this band is probably the result of aggregate formation. Nevertheless, the possibility that the $75 \mathrm{kDa}$ band may represent Eis covalently bound to a mammalian protein needs to be further investigated.
These findings are consistent with the reports of detection of other mycobacterial proteins and lipids in vesicles released into the tissue culture supernatant by $M$. tuberculosisinfected macrophages (Beatty et al., 2001, 2000; Rhoades et al., 2003). The steps utilized to obtain the vesicular pellet resulted in a tissue culture supernatant fraction devoid of any host cellular structural material. Western blot analysis of a concentrated aliquot of this fraction detected the presence of the Eis protein (Fig. 3, lane 4), the source of which may be the degradation of vesicles containing mycobacterial proteins in the culture supernatant or possibly due to exocytic release of protein from M. tuberculosis-infected macrophages.

Viability of infected macrophages was determined using an LDH release assay (Promega) to ensure that the release of Eis and vesicles containing Eis into the tissue culture supernatant was not due to lysis of infected macrophages. Based on $\mathrm{LDH}$ release, it was determined that macrophage viability was $>90 \%$ at time of harvest $(48 \mathrm{~h})$.

\section{Cytokine release by human monocytes in response to stimulation with recombinant Eis}

To evaluate cytokine production in primary human monocytes in response to recombinant Eis or PPD antigen (an M. tuberculosis antigen preparation commonly used in such immunological studies), assays were performed to detect TNF- $\alpha$ and IL-10 secretion by ELISA. These cytokines are known to play a significant role in the pro- and antiinflammatory components respectively of the immune response to M. tuberculosis infection (Flynn et al., 1995; Gong et al., 1996). Mycobacterial proteins/lipids released from the phagosome of $M$. tuberculosis-infected macrophages have also been shown to modulate levels of cytokine secretion during infection, to the advantage of the pathogen (Gil et al., 2004; Placido et al., 1997; Rivera-Marrero et al., 2004).

Experiments using human monocytes from three healthy tuberculin reactors showed that TNF- $\alpha$ and IL-10 were not produced in freshly isolated cells but were detectable $3 \mathrm{~h}$ after and peaked from 18-24 h (for TNF- $\alpha$ ) and $48 \mathrm{~h}$ (for IL-10) after stimulation with recombinant Eis or PPD antigen (data not shown). Therefore, the $18 \mathrm{~h}$ and $48 \mathrm{~h}$ time points were used to study the levels of TNF- $\alpha$ and IL-10 production, respectively, after stimulation with the Eis or PPD antigens of M. tuberculosis. As shown in Fig. 4(a), Eisinduced TNF- $\alpha$ production was increased in human monocytes in a dose-dependent manner. However, Eis elicited significantly lower amounts of TNF- $\alpha$ at 5 and $10 \mu \mathrm{g} \mathrm{ml}^{-1}$, when compared with the same concentrations of the PPD antigen $(P<0.05$; Fig. 4a). Stimulation of human monocytes with $10 \mathrm{ng}$ LPS (positive control) resulted in the secretion of TNF- $\alpha\left(1000-2500 \mathrm{pg} \mathrm{ml}^{-1}\right)$.

As shown in Fig. 4(b), significant dose-dependent IL-10 production was observed by human monocytes after in vitro stimulation with the Eis protein. In contrast to the findings 

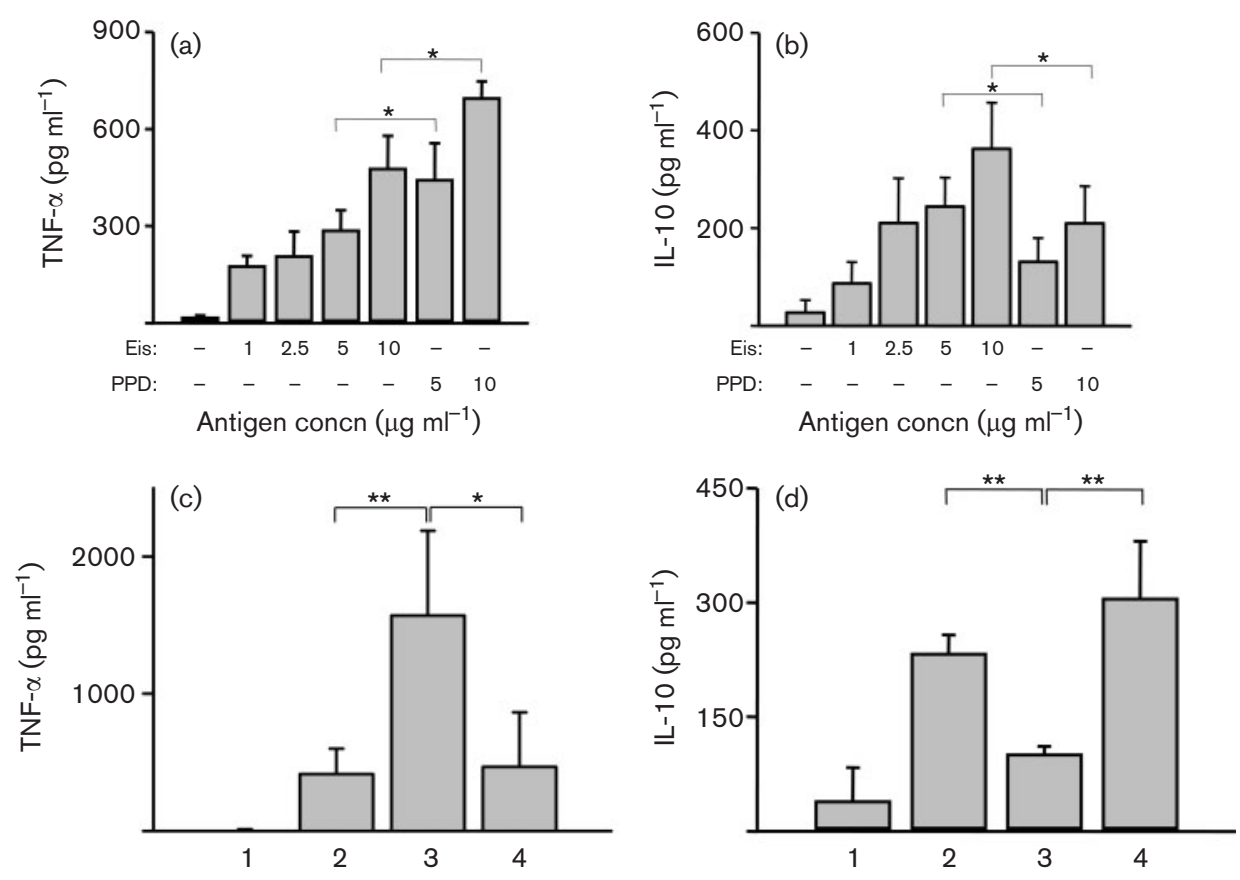

\begin{abstract}
Fig. 4. Cytokine secretion by human monocytes in response to Eis. Monocytes were isolated from tuberculin-positive healthy donors and either left untreated or stimulated with increasing amounts of PPD and Eis antigen. (a) TNF- $\alpha$ concentration was measured by ELISA in the cell culture supernatants harvested at $18 \mathrm{~h}$ following stimulation with each antigen and expressed as the mean $\pm S E$ of three independent experiments. (b) IL-10 concentration was measured by ELISA in the cell culture supernatants harvested at $48 \mathrm{~h}$ following stimulation with each antigen and expressed as the mean \pm SD of three independent experiments. $n=3$ for each experiment. ${ }^{*} P<0.05$. (c) Monocytes from healthy tuberculin-positive donors were harvested and either not infected (1), or infected with M. tuberculosis H37Rv wild-type (2), Deis (3) and complemented strains (4) at an m.o.i. of 1. Culture supernatants were harvested $18 \mathrm{~h}$ after infection to determine levels of cytokine production. TNF- $\alpha$ levels in the culture supernatants were monitored by ELISA. A representative experiment is shown, $n=3$. (d) IL-10 secretion by infected monocytes from healthy donors as described for (c). IL-10 levels in the supernatants were monitored by ELISA. A representative experiment is shown in (b), $n=3 .{ }^{\star} P<0.05 ;{ }^{\star}{ }^{*} P<0.01$.
\end{abstract}

for TNF- $\alpha$, the IL-10 production induced by Eis protein at 5 and $10 \mu \mathrm{g} \mathrm{ml}^{-1}$ was higher $(P<0.05)$ than that induced by the PPD antigen at the same concentrations (Fig. $4 \mathrm{~b}$ ). Stimulation of human monocytes with $10 \mathrm{ng}$ LPS (positive control) resulted in the increased secretion of IL-10 $\left(2000-4000 \mathrm{pg} \mathrm{ml}^{-1}\right)$. Recombinant protein preparations were tested to ensure absence of any contaminating endotoxins.

\section{Cytokine release by human monocytes in response to infection with $M$. tuberculosis H37Rv wild-type, $\Delta$ eis and complemented strains}

Next, TNF- $\alpha$ and IL-10 secretion by human monocytes infected with wild-type $M$. tuberculosis $\mathrm{H} 37 \mathrm{Rv}, \Delta e i$ s and eis complemented strains at an m.o.i. of 1 were evaluated. As shown in Fig. 4(c), TNF- $\alpha$ production by human monocytes infected with the H37Rv $\Delta$ eis was significantly greater than those infected with wild-type $\mathrm{H} 37 \mathrm{Rv}(P<0.01)$ or the complemented strain $(P<0.05)$. Although the mean TNF- $\alpha$ production by $M$. tuberculosis-infected monocytes increased slightly with bacterial load, $\mathrm{H} 37 \mathrm{Rv}$ wild-type infected monocytes still secreted significantly less TNF- $\alpha$ than those infected with the $\Delta e i s$ strain at an m.o.i. of 10 (data not shown).

In contrast to TNF- $\alpha$, IL-10 secretion by $H 37 \mathrm{Rv} \Delta$ eisinfected monocytes was significantly lower than induction by H37Rv wild-type $(P<0.01)$ or eis-complemented strains $(P<0.01)$ (Fig. 4 d). With increased bacterial load (m.o.i. of $10)$, the H37Rv $\Delta$ eis-infected monocytes secreted significantly less IL-10 than those infected with wild-type or complemented strains (data not shown). These results show that strain $\mathrm{H} 37 \mathrm{Rv} \Delta$ eis induced more TNF- $\alpha$ but less IL-10 production in primary human monocytes than either $\mathrm{H} 37 \mathrm{Rv}$ wild-type or complemented strains.

\section{DISCUSSION}

The eis gene of M. tuberculosis enhances the intracellular survival of M. smegmatis (Wei et al., 2000). However, its function in the virulence of $M$. tuberculosis has not yet been elucidated. No significant difference was noted in the 
survival of $M$. tuberculosis $\mathrm{H} 37 \mathrm{Rv}$ wild-type, $\Delta$ eis mutant and complemented strains in either in vitro or in vivo models (Fig. 1). However, Barczak et al. (2005) demonstrated that there was no significant difference in the growth of the hypovirulent CDC 1551 and the hypervirulent HN878 strains of $M$. tuberculosis in prestimulated macrophages. They similarly noted no difference in the mycobacterial loads of the lungs of mice infected with either strain. Instead the difference between the two strains lay in the growth kinetics and mouse survival (Barczak et al., 2005). Further studies are required to determine whether the same is true of strains deficient in eis. It is possible that the role of eis in the pathogenesis of $M$. tuberculosis may differ significantly from the phenotype observed in M. smegmatis (Wei et al., 2000), a surrogate host for this M. tuberculosis-derived gene, thus explaining the lack of an intracellular survival phenotype in the current study.

The discovery that eis is expressed during infection of activated human macrophages by clinical strains of $M$. tuberculosis has been confirmed by others (Cappelli et al., 2001). In these present studies, RT-PCR indicated that eis is constitutively expressed by the M. tuberculosis H37Ra strain whether in culture media or in macrophages (data not shown). This idea is corroborated by studies by Dahl et al. (2005) showing that eis is negatively regulated by the stringent response regulator $R e l_{\mathrm{Mtb}}$ so as to maintain constant levels of expression during starvation conditions. On the other hand, Wu et al. (2005) have demonstrated that the expression of eis was enhanced 12-fold in the clinical strain M. tuberculosis 210 as compared to the H37Rv strain when grown in a human monocyte cell line, whereas the expression was the same when the strains were grown in vitro. They also showed that SigA binds to the promoter region of eis and is responsible for the upregulation of eis expression in strain 210. They concluded that eis may contribute to the enhanced growth of the 210 strain in human macrophages. Morris et al. (2005) have also recently demonstrated that eis is a component of the whiB7 regulon, whose expression is upregulated by exposure to sublethal concentrations of antibiotics or fatty acids such as would be encountered within the host macrophage during infection. These reports in conjunction with the data presented here point to a significant but not yet clearly defined role for eis in the pathogenesis of $M$. tuberculosis.

Multiple sources have reported on the release of mycobacterial constituents from infected macrophages into the culture medium followed by their uptake into uninfected bystander cells (Beatty \& Russell, 2000; Beatty et al., 2001; Rhoades et al., 2003). Our studies using IF of MtbRa/gfpinfected U-937 macrophages demonstrate the production and presence of Eis in the macrophage cytoplasm from $4 \mathrm{~h}$ to $96 \mathrm{~h}$ post-infection (Fig. $2 \mathrm{c}-\mathrm{h}$ ). Furthermore, macrophage lysate fractions, purged of infecting bacteria, demonstrated the presence of the Eis protein by Western blot analysis (Fig. 3, lane 2). The presence of Eis in the extravesicular fraction and the tissue culture supernatant of infected macrophages (Fig. 3, lanes 3 and 4) places it in the company of other known mycobacterial effectors of virulence such as lipoarabinomannan (LAM) (Beatty et al., 2000). These mycobacterial protein/lipid-containing vesicles appear to be taken up by uninfected macrophages, which accounts for the presence of mycobacterial components within host cells in the absence of bacteria (Beatty et al., 2000). The release of Eis into the culture supernatant by infected macrophages, either in vesicles or in native form, followed by its uptake into uninfected macrophages (Fig. $2 \mathrm{~g}, \mathrm{~h}$ ), is the most likely explanation for its presence in these cells. Further work needs to be done to determine the localization of secreted Eis within the macrophage and examine its significance.

The critical importance of TNF- $\alpha$ in anti-mycobacterial defence is well established in mice and humans (Flynn et al., 1995; Keane et al., 2001). TNF- $\alpha$ helps prevent the reactivation of persistent $\mathrm{TB}$ and limits the pathological response of the host. The concept that $M$. tuberculosis plays a role in modulation of the TNF- $\alpha / \mathrm{IL}-10$ axis is supported by our data showing that Eis modulates TNF- $\alpha$ and IL-10 secretion by human monocytes in response to infection by M. tuberculosis (Fig. 4c, d). Because purified Eis elicits higher levels of IL-10 than TNF- $\alpha$ secretion, as compared to PPD (Fig. 4a, b), we examined the differences in cytokine secretion in response to infection with viable $M$. tuberculosis $\mathrm{H} 37 \mathrm{Rv}$ wild-type, $\Delta e$ is and eis-complemented strains. In contrast to $\mathrm{H} 37 \mathrm{Rv}$ and the complemented strain, the $\Delta$ eis mutant induced significantly higher levels of TNF- $\alpha$ and lower levels of IL-10 in human monocytes (Fig. 4c, d). This provides powerful evidence that $M$. tuberculosis utilizes Eis to manipulate the host TNF- $\alpha /$ IL-10 axis to its advantage by inducing a state of localized immunosuppression in the host.

IL-10 is thought to play an important role in the regulation of host antimicrobial immunity. The induction of downregulatory cytokines, such as IL-10 and transforming growth factor- $\beta$, can inhibit interferon (IFN) $-\gamma$ production by T cells and block or inhibit macrophage activation and apoptosis (Flesch et al., 1994; Othieno et al., 1999). It is widely known that in alveolar macrophages, apoptosis is a common defence mechanism against $M$. tuberculosis infection via a TNF- $\alpha$-mediated pathway (Placido et al., 1997). Monocytes from patients with active TB when compared to those from healthy donors are not only likely to secrete more IL-10 than TNF- $\alpha$ in response to stimulation with PPD, but they are also more likely to undergo necrosis than apoptosis (Gil et al., 2004). Utilizing the slowly progressive primary tuberculosis (SPTB) mouse model, Abebe et al. (2006) have demonstrated a 20 -fold increase in the expression levels of IL-10 in the lungs of mice with progressive tuberculosis as compared to mice with latent tuberculosis. Increased IL-10, but decreased TNF- $\alpha$ production driven by Eis, and possibly other M. tuberculosis antigens, may contribute to the intracellular survival of the pathogen by suppressing host cell apoptosis during mycobacterial infection. It is quite likely that the modulation of TNF- $\alpha$ and IL-10 secretion by 
Eis will have a further effect on the levels of other cytokines due to their roles as prominent components of the pro- and anti-inflammatory immune response. Immunohistochemical analysis of $M$. tuberculosis-infected tissues for secreted Eis as well as altered cytokine expression may shed light on the role of eis in the pathogenesis of M. tuberculosis.

Bioinformatic analysis suggests that Eis is an acetyltransferase of the family of GCN5-related $\mathrm{N}$-acetyltransferases. 3DPSSM fold analysis shows that the predicted structure of Eis bears significant similarity to the structures of known members of the GNAT superfamily (Table 3 ) and also shares the conserved residues that are characteristic of this family. This family of proteins is involved in a wide variety of activities ranging from transcriptional activation by histone acetyltransferases to antibiotic resistance by aminoglycoside acetyltransferases. The lack of sequence homology among members of this family makes classification of possible new members difficult unless 3D structural data are available (Neuwald \& Landsman, 1997). In the absence of candidate substrates, it is extremely difficult to demonstrate whether the Eis protein possesses functional acetyltransferase activity. Current efforts are being directed towards identification of possible substrates and resolution of the crystal structure of the Eis protein, which may provide concrete evidence as to the putative acetyltransferase activity of Eis and also offer direction as to the identity of possible substrates.

The absence of an observable intracellular survival phenotype for eis, while confounding, should not detract from the significant nature of the other findings presented here. Recent reports regarding the role of eis in clinical strains of $M$. tuberculosis indicate that further studies should be conducted using these strains. The release of Eis into the cytoplasm of infected macrophages and its apparent role in the modulation of cytokine secretion in these important host immune cells, essential for defence against tuberculosis infection, raises many interesting questions as to the mechanism(s) whereby this modulation is achieved. Perhaps Eis acetylates histones as other acetyltransferases do (Brownell \& Allis, 1995) in the macrophage nucleus, resulting in reduced binding to DNA and enhanced transcription and translation of IL- 10 . Alternatively, Eis may act directly on mammalian cell transcription factors and alter the response of the host cell to infection as described for other acetyltransferases (Sterner \& Berger, 2000). Eis, by one of these mechanisms, may directly affect macrophage cytokine production via activity in the host cell nucleus. Whether Eis may actually enter and localize in the macrophage nucleus is unknown and is presently under investigation. The data presented here point toward a significant and novel role for eis as yet another virulence factor in the pathogenesis of $M$. tuberculosis that warrants further study.

\section{ACKNOWLEDGEMENTS}

We thank James Moulder, Janet Hatt, Amy Windley and Christopher Alteri for invaluable assistance and technical suggestions. This work was supported in part by National Institutes of Health grant AI4553701A2 to R. L.F. and Grant R042 0040001002202004 from the Korea Research Foundation for the 2004 program year to E. K. J.

\section{REFERENCES}

Abebe, F., Mustafa, T., Nerland, A. H. \& Bjune, G. A. (2006). Cytokine profile during latent and slowly progressive primary tuberculosis: a possible role for interleukin-15 in mediating clinical disease. Clin Exp Immunol 143, 180-192.

Altschul, S. F., Gish, W., Miller, W., Myers, E. W. \& Lipman, D. J. (1990). Basic local alignment search tool. J Mol Biol 215, 403-410.

Barczak, A. K., Domenech, P., Boshoff, H. I., Reed, M. B., Manca, C., Kaplan, G. \& Barry, C. E., 3rd (2005). In vivo phenotypic dominance in mouse mixed infections with Mycobacterium tuberculosis clinical isolates. J Infect Dis 192, 600-606.

Barker, L. P., Brooks, D. M. \& Small, P. L. (1998). The identification of Mycobacterium marinum genes differentially expressed in macrophage phagosomes using promoter fusions to green fluorescent protein. Mol Microbiol 29, 1167-1177.

Barnes, P. F., Chatterjee, D., Abrams, J. S., Lu, S., Wang, E., Yamamura, M., Brennan, P. J. \& Modlin, R. L. (1992). Cytokine production induced by Mycobacterium tuberculosis lipoarabinomannan. Relationship to chemical structure. J Immunol 149, 541-547.

Beatty, W. L. \& Russell, D. G. (2000). Identification of mycobacterial surface proteins released into subcellular compartments of infected macrophages. Infect Immun 68, 6997-7002.

Beatty, W. L., Rhoades, E. R., Ullrich, H. J., Chatterjee, D., Heuser, J. E. \& Russell, D. G. (2000). Trafficking and release of mycobacterial lipids from infected macrophages. Traffic 1, 235-247.

Beatty, W. L., Ullrich, H. J. \& Russell, D. G. (2001). Mycobacterial surface moieties are released from infected macrophages by a constitutive exocytic event. Eur J Cell Biol 80, 31-40.

Boshoff, H. I., Reed, M. B., Barry, C. E., 3rd \& Mizrahi, V. (2003). DnaE2 polymerase contributes to in vivo survival and the emergence of drug resistance in Mycobacterium tuberculosis. Cell 113, 183-193.

Brownell, J. E. \& Allis, C. D. (1995). An activity gel assay detects a single, catalytically active histone acetyltransferase subunit in Tetrahymena macronuclei. Proc Natl Acad Sci U S A 92, 6364-6368.

Cappelli, G., Volpe, P., Sanduzzi, A., Sacchi, A., Colizzi, V. \& Mariani, F. (2001). Human macrophage gamma interferon decreases gene expression but not replication of Mycobacterium tuberculosis: analysis of the host-pathogen reciprocal influence on transcription in a comparison of strains $\mathrm{H} 37 \mathrm{Rv}$ and CMT97. Infect Immun 69, 7262-7270.

Chakraborty, P., Sturgill-Koszycki, S. \& Russell, D. G. (1994). Isolation and characterization of pathogen-containing phagosomes. Methods Cell Biol 45, 261-276.

Chan, J., Fan, X. D., Hunter, S. W., Brennan, P. J. \& Bloom, B. R. (1991). Lipoarabinomannan, a possible virulence factor involved in persistence of Mycobacterium tuberculosis within macrophages. Infect Immun 59, 1755-1761.

Corbett, E. L., Watt, C. J., Walker, N., Maher, D., Williams, B. G., Raviglione, M. C. \& Dye, C. (2003). The growing burden of tuberculosis: global trends and interactions with the HIV epidemic. Arch Intern Med 163, 1009-1021.

Dahl, J. L., Wei, J., Moulder, J. W., Laal, S. \& Friedman, R. L. (2001). Subcellular localization of the intracellular survival-enhancing Eis protein of Mycobacterium tuberculosis. Infect Immun 69, 4295-4302.

Dahl, J. L., Kraus, C. N., Boshoff, H. I., Doan, B., Foley, K., Avarbock, D., Kaplan, G., Mizrahi, V., Rubin, H. \& Barry, C. E., 3rd (2003). The 
role of RelMtb-mediated adaptation to stationary phase in long-term persistence of Mycobacterium tuberculosis in mice. Proc Natl Acad Sci U S A 100, 10026-10031.

Dahl, J. L., Arora, K., Boshoff, H. I., Whiteford, D. C., Pacheco, S. A., Walsh, O. J., Lau-Bonilla, D., Davis, W. B. \& Garza, A. G. (2005). The relA homolog of Mycobacterium smegmatis affects cell appearance, viability, and gene expression. J Bacteriol 187, 2439-2447.

D’Andrea, A., Aste-Amezaga, M., Valiante, N. M., Ma, X., Kubin, M. \& Trinchieri, G. (1993). Interleukin 10 (IL-10) inhibits human lymphocyte interferon gamma-production by suppressing natural killer cell stimulatory factor/IL-12 synthesis in accessory cells. $J$ Exp Med 178, 1041-1048.

de Waal Malefyt, R., Abrams, J., Bennett, B., Figdor, C. G. \& de Vries, J. E. (1991). Interleukin 10 (IL-10) inhibits cytokine synthesis by human monocytes: an autoregulatory role of IL-10 produced by monocytes. J Exp Med 174, 1209-1220.

Dubnau, E., Fontan, P., Manganelli, R., Soares-Appel, S. \& Smith, I. (2002). Mycobacterium tuberculosis genes induced during infection of human macrophages. Infect Immun 70, 2787-2795.

Dyda, F., Klein, D. C. \& Hickman, A. B. (2000). GCN5-related $N$ acetyltransferases: a structural overview. Annu Rev Biophys Biomol Struct 29, 81-103.

Flesch, I. E., Hess, J. H., Oswald, I. P. \& Kaufmann, S. H. (1994). Growth inhibition of Mycobacterium bovis by IFN-gamma stimulated macrophages: regulation by endogenous tumor necrosis factor-alpha and by IL-10. Int Immunol 6, 693-700.

Flynn, J. L., Goldstein, M. M., Chan, J., Triebold, K. J., Pfeffer, K., Lowenstein, C. J., Schreiber, R., Mak, T. W. \& Bloom, B. R. (1995). Tumor necrosis factor-alpha is required in the protective immune response against Mycobacterium tuberculosis in mice. Immunity 2, 561-572.

Fratti, R. A., Chua, J., Vergne, I. \& Deretic, V. (2003). Mycobacterium tuberculosis glycosylated phosphatidylinositol causes phagosome maturation arrest. Proc Natl Acad Sci U S A 100, 5437-5442.

Fulton, S. A., Johnsen, J. M., Wolf, S. F., Sieburth, D. S. \& Boom, W. H. (1996). Interleukin-12 production by human monocytes infected with Mycobacterium tuberculosis: role of phagocytosis. Infect Immun 64, 2523-2531.

Gil, D. P., Leon, L. G., Correa, L. I., Maya, J. R., Paris, S. C., Garcia, L. F. \& Rojas, M. (2004). Differential induction of apoptosis and necrosis in monocytes from patients with tuberculosis and healthy control subjects. J Infect Dis 189, 2120-2128.

Gong, J. H., Zhang, M., Modlin, R. L., Linsley, P. S., Iyer, D., Lin, Y. \& Barnes, P. F. (1996). Interleukin-10 downregulates Mycobacterium tuberculosis-induced Th1 responses and CTLA-4 expression. Infect Immun 64, 913-918.

Keane, J., Gershon, S., Wise, R. P., Mirabile-Levens, E., Kasznica, J., Schwieterman, W. D., Siegel, J. N. \& Braun, M. M. (2001). Tuberculosis associated with infliximab, a tumor necrosis factor alpha-neutralizing agent. $N$ Engl J Med 345, 1098-1104.

Kelley, L. A., MacCallum, R. M. \& Sternberg, M. J. (2000). Enhanced genome annotation using structural profiles in the program $3 \mathrm{D}$ PSSM. J Mol Biol 299, 499-520.

Lee, J. S., Song, C. H., Lim, J. H., Kim, H. J., Park, J. K., Paik, T. H., Kim, C. H., Kong, S. J., Shon, M. H. \& other authors (2003a). The production of tumour necrosis factor-alpha is decreased in peripheral blood mononuclear cells from multidrug-resistant tuberculosis patients following stimulation with the $30-\mathrm{kDa}$ antigen of Mycobacterium tuberculosis. Clin Exp Immunol 132, 443-449.

Lee, J. S., Song, C. H., Kim, C. H., Kong, S. J., Shon, M. H., Suhr, J. W., Jung, S. S., Lim, J. H., Kim, H. J. \& other authors (2003b). Depressed interleukin-12 production by peripheral blood mononuclear cells after in vitro stimulation with the $30-\mathrm{kDa}$ antigen in recurrent pulmonary tuberculosis patients. Med Microbiol Immunol 192, 61-69.

Marchler-Bauer, A. \& Bryant, S. H. (2004). CD-Search: protein domain annotations on the fly. Nucleic Acids Res 32, W327-W331.

Morris, R. P., Nguyen, L., Gatfield, J., Visconti, K., Nguyen, K., Schnappinger, D., Ehrt, S., Liu, Y., Heifets, L. \& other authors (2005). Ancestral antibiotic resistance in Mycobacterium tuberculosis. Proc Natl Acad Sci U S A 102, 12200-12205.

Neuwald, A. F. \& Landsman, D. (1997). GCN5-related histone $\mathrm{N}$ acetyltransferases belong to a diverse superfamily that includes the yeast SPT10 protein. Trends Biochem Sci 22, 154-155.

Nilsson, K. \& Sundstrom, C. (1974). Establishment and characteristics of two unique cell lines from patients with lymphosarcoma. Int J Cancer 13, 808-823.

Othieno, C., Hirsch, C. S., Hamilton, B. D., Wilkinson, K., Ellner, J. J. \& Toossi, Z. (1999). Interaction of Mycobacterium tuberculosisinduced transforming growth factor beta 1 and interleukin-10. Infect Immun 67, 5730-5735.

Pelicic, V., Jackson, M., Reyrat, J. M., Jacobs, W. R., Jr, Gicquel, B. \& Guilhot, C. (1997). Efficient allelic exchange and transposon mutagenesis in Mycobacterium tuberculosis. Proc Natl Acad Sci U S A 94, 10955-10960.

Placido, R., Mancino, G., Amendola, A., Mariani, F., Vendetti, S., Piacentini, M., Sanduzzi, A., Bocchino, M. L., Zembala, M. \& Colizzi, V. (1997). Apoptosis of human monocytes/macrophages in Mycobacterium tuberculosis infection. J Pathol 181, 31-38.

Rhoades, E., Hsu, F., Torrelles, J. B., Turk, J., Chatterjee, D. \& Russell, D. G. (2003). Identification and macrophage-activating activity of glycolipids released from intracellular Mycobacterium bovis BCG. Mol Microbiol 48, 875-888.

Rivera-Marrero, C. A., Stewart, J., Shafer, W. M. \& Roman, J. (2004). The down-regulation of cathepsin G in THP-1 monocytes after infection with Mycobacterium tuberculosis is associated with increased intracellular survival of bacilli. Infect Immun 72, 5712-5721.

Sambrook, J. E., Fritsch, E. F. \& Maniatis, T. (1989). Molecular Cloning: a Laboratory Manual. Cold Spring Harbor, NY: Cold Spring Harbor Laboratory.

Saviola, B., Woolwine, S. C. \& Bishai, W. R. (2003). Isolation of acidinducible genes of Mycobacterium tuberculosis with the use of recombinase-based in vivo expression technology. Infect Immun 71, 1379-1388.

Song, C. H., Lee, J. S., Lee, S. H., Lim, K., Kim, H. J., Park, J. K., Paik, T. H. \& Jo, E. K. (2003). Role of mitogen-activated protein kinase pathways in the production of tumor necrosis factor-alpha, interleukin-10, and monocyte chemotactic protein-1 by Mycobacterium tuberculosis H37Rv-infected human monocytes. J Clin Immunol 23, 194-201.

Sterner, D. E. \& Berger, S. L. (2000). Acetylation of histones and transcription-related factors. Microbiol Mol Biol Rev 64, 435-459.

Stover, C. K., de la Cruz, V. F., Fuerst, T. R., Burlein, J. E., Benson, L. A., Bennett, L. T., Bansal, G. P., Young, J. F., Lee, M. H. \& other authors (1991). New use of BCG for recombinant vaccines. Nature 351, 456-460.

Wallis, R. S., Amir-Tahmasseb, M. \& Ellner, J. J. (1990). Induction of interleukin 1 and tumor necrosis factor by mycobacterial proteins: the monocyte western blot. Proc Natl Acad Sci U S A 87, 3348-3352.

Wei, J., Dahl, J. L., Moulder, J. W., Roberts, E. A., O’Gaora, P., Young, D. B. \& Friedman, R. L. (2000). Identification of a Mycobacterium tuberculosis gene that enhances mycobacterial survival in macrophages. J Bacteriol 182, 377-384.

Wu, S., Howard, S. T., Samten, B., Rodrigue, S., Gaudreau, L. \& Barnes, P. F. (2005). The principal sigma factor siga upregulates 
expression of the eis gene in a clinical Mycobacterium tuberculosis Beijing strain. In Abstracts of the Keystone Symposium on Tuberculosis, abstract no. 3118.

Xu, S., Cooper, A., Sturgill-Koszycki, S., van Heyningen, T., Chatterjee, D., Orme, I., Allen, P. \& Russell, D. G. (1994).
Intracellular trafficking in Mycobacterium tuberculosis and Mycobacterium avium-infected macrophages. J Immunol 153, 2568-2578.

Edited by: G. S. Besra 